\title{
Empresas familiares: Conceptos, teorías y estructuras
}

\begin{abstract}
FECHA DE RECEPCIÓN: 27 de julio
FECHA DE APROBACIÓN: 26 de agosto

pp. 149-158
\end{abstract}

DOI: http://dx.doi.org/10.21158/01208160.n81.2016.1555

\section{Resumen}

Este documento presenta aspectos conceptuales, teóricos, históricos y estructurales, de las empresas familiares. Se destaca el rol que desempeñan en materia de generación de empleo y riqueza. También, se analizan sus sistemas de gobierno corporativo, los cuales desembocan en la temática de continuidad generacional; la continuidad es entendida como una de las principales dificultades de estas organizaciones, debido a que sus directivos y/o propietarios no logran separar el papel del núcleo familiar y las decisiones organizacionales de las compañías. En este sentido, las estrategias de internacionalización y profesionalización representan el camino para garantizar la permanencia de las empresas familiares.

\section{Palabras clave}

Empresa familiar, gobierno corporativo, internacionalización, profesionalización, emprendimientos, generación de empleo.

\section{Raúl Francisco Quejada Pérez}

PhD.(c), Universidad de Zulia, Venezuela; magíster en Ciencias Económicas, Universidad Nacional de Colombia y economista, Universidad de Cartagena. Docente de la Facultad de Ciencias Económicas, Universidad de Cartagena; director del Grupo Investigación en Mercado Laboral.

\section{Jorge Nelson Ávila Gutiérrez}

Titular de postdoctorados y doctorados en educación superior, economía, gerencia, ciencia política, finanzas internacionales, Universidad del Zulia, Venezuela. Jefe de Misiones Inteligenciales del Sistema de las Naciones Unidas. 


\title{
Family- owned Companies: Definition, Theories and Structures
}

\begin{abstract}
Abstrac. This article describes conceptual, theoretical, historical and structural aspects of family- owned companies. Their role in terms of the generation of employment and wealth is also shown. Additionally, different systems of corporative governance are analysed, which link with the topic of generation continuity. Such continuity is understood as one of the main obstacles that these companies face as their executive boards and owners cannot leave apart the role of the family and the company decisions to take. Consequently, the strategies of internationalization and professionalization pave the way to facilitate the existence of family- owned companies.
\end{abstract}

Key words. Family- owned companies, corporate governance, internationalization, professionalization, entrepreneurships, employment generation.

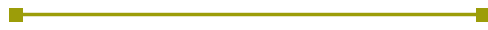 \\ Entreprises familiales: Concepts, théories et structures}

Resumé. Ce document présente les aspects conceptuels, théoriques, historiques etstructurels des entreprises familiales et insiste sur leur rôle en termes de création d'emploi et de richesse. L'article analyse également les systèmes de gouvernance corporative débouchant sur une thématique de continuité générationnelle. La continuité familiale semble être l'une des difficultés principales que rencontrent ces organisations du fait que leurs dirigeants et/ou propriétaires ne réussissent pas toujours à séparer le rôle joué par le noyau familial des décisions entrepreneuriales des compagnies. Les stratégies d'internationalisation et de professionnalisation des entreprises familiales semblent donc être la garantie de la pérennité de ces organisations.

Mots clefs. Entreprise familiale, gouvernance corporative, internationalisation, professionnalisation, initiatives, création d'emplois.

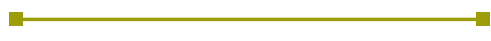 \\ Empresas familiares: Conceitos, teorias e estruturas}

Resumo. Este documento apresenta aspetos conceptuais, teóricos, históricos e estruturais das empresas familiares. Destaca-se o rol que desempenham na matéria de geração de emprego e riqueza. Também se analisam seus sistemas de governo corporativo, os quais desembocam na temática de continuidade geracional. A continuidade é entendida como uma das principais dificuldades destas organizações, devido a que seus diretivos elou proprietários, não conseguem separar o papel do núcleo familiar e as decisões organizacionais das empresas. Neste sentido, as estratégias de internacionalização e profissionalização representam o caminho para garantir a permanência das empresas familiares.

Palabras chave. Empresa familiar, governo corporativo, internacionalização, profissionalização, empreendimentos, geração de empregos. 


\section{Introducción}

T a temática de las empresas familiares Japarece en el contexto actual de los tópicos académicos debido a la importancia que este tipo de compañías tienen dentro de las estructuras de producción de las economías, tanto desarrolladas como en vías de desarrollo. Su importancia radica en el poder generador de empleos que representa, y en el espíritu emprendedor que personifican sus fundadores. No obstante, también se reconocen cuellos de botella dentro de su desempeño, debido al difícil sostenimiento en el tiempo, a causa de las denominadas crisis generacionales. En este sentido, las empresas familiares deben estructurar y establecer estrategias de mitigación de este flagelo, a través de la internacionalización y profesionalización de sus recursos humanos, administrativos y financieros.

A continuación, se presentan los principales elementos conceptuales, históricos y estructurales de las denominadas empresas familiares.

\section{Marco conceptual, teórico e histórico}

T n una época caracterizada por la creciente Capertura de las economías nacionales y el predominio de las grandes empresas transnacionales, la empresa familiar (EF) tiende a ser percibida como parte del pasado, al concebirse como una modalidad de organización empresarial que poco tiene que ver con la era de la globalización. No obstante, hoy por hoy, las empresas familiares continúan siendo muy importantes en la economía de cualquier país. Datos de la Cooperación Financiera Internacional del Banco Mundial (IFC, Banco Mundial, 2011), muestran que en Estados Unidos existen 12 millones de empresas familiares que proveen el $59 \%$ de los puestos de trabajo y generan el $78 \%$ de los nuevos empleos. En España, por su parte, las empresas familiares representan un $71 \%$ del total de empresas. Esta tendencia es la constante a nivel mundial.

De acuerdo con Leach (2002), en Bañegil Palacios, Barroso Martínez y Tato Jiménez (2011), la empresa familiar es una organización social de gran complejidad, donde conviven la empresa y la familia, entes que se desempeñan como dos subsistemas superpuestos, interdependientes y generadores de conflicto.

Operativamente, de acuerdo con Lozano Posso (2000), la empresa familiar también puede definirse como un tipo de unidad empresarial que suele operar a partir de la disponibilidad de capital y trabajo de origen familiar o doméstico, y en la cual las relaciones laborales presentan elementos diferenciadores con respecto a otro tipo de organizaciones empresariales. Soto Maciel (2013), la define como aquella compañía donde la mayoría de los votos está en manos de la familia controladora, incluyendo al fundador, el cual busca heredar la empresa a sus descendientes, obligándolos a conservar las raíces y los valores que permitan mantener la unidad familiar a través del patrimonio (Steckerl, 2006).

Es importante anotar, que dentro del devenir histórico, las empresas familiares han 
constituido la forma de organización empresarial más antigua, siendo en la mayoría de los casos, la base de los procesos de desarrollo que suceden a las grandes crisis estructurales (IFC. Banco Mundial, 2011). Para Soto Maciel (2013), las primeras formas de organización de la actividad comercial e industrial constituyeron una extensión del sistema familiar. En el campo de la investigación académica, los países industrializados han sido los primeros en valorar el papel económico de la empresa familiar, al considerarla parte fundamental de las estructuras debido a su preponderante papel en la generación de riqueza nacional.

A nivel teórico, a pesar de que pueden identificarse trabajos iniciales hacia mediados del

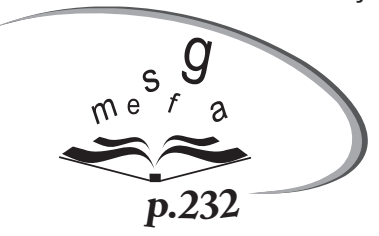

siglo pasado, es en la década de los 80 que el estudio de la temática se intensifica con Schein (1983), Dyer (1988) y Handler (1989, en Soto Maciel, 2013). No obstante, en la actualidad, la noción de empresa familiar carece aún de consenso a pesar de la preferencia de los investigadores por retomar el modelo de los tres círculos de Tagiuri y Davis (1982), donde condicionan su existencia a la interconexión de tres subsistemas: la propiedad, la familia y la empresa. De acuerdo con Croutsche y Ganidis (2008), esta heterogeneidad conceptual se relaciona, principalmente, con la dificultad para fijar los límites fronterizos entre el lazo familiar, el nivel de control y el tiempo de posesión de la empresa (Soto Maciel, 2013).

A continuación se presentan con los principales autores que han tratado el concepto de empresa familiar en la última década. Es de anotar que los conceptos se encuentran influenciados por la teoría del triple círculo (Tabla1).

Tabla 1. Conceptos sobre empresa familiar.

\begin{tabular}{|c|l|}
\hline Hoshino (2004) & Empresas poseídas y controladas por familias o descendientes del fundador. \\
\hline De la Garza (2003) & Empresa donde los sistemas de familia y empresa se entrelazan. \\
\hline Belausteguigoitia (2007) & $\begin{array}{l}\text { Empresa poseída o controlada por una familia y en la que los miembros se } \\
\text { encuentran relacionados con el negocio. }\end{array}$ \\
\hline Navarrete (2008) & $\begin{array}{l}\text { Empresa cuya propiedad está en manos de uno o más miembros de un } \\
\text { grupo familiar y su dirección y sucesión tendrá influencia familiar. }\end{array}$ \\
\hline Esparza (2009) & $\begin{array}{l}\text { Empresa donde más del } 50 \text { \% del capital es propiedad de la familia y los } \\
\text { puestos de dirección los ocupa al menos un representante familiar. }\end{array}$ \\
\hline Flórez (2010) & Empresa que se identifica con al menos dos generaciones de la familia. \\
\hline Suárez (2010) & Organización con modelo tridimensional: familia, empresa y propiedad. \\
\hline Ramírez y Fonseca (2010) & $\begin{array}{l}\text { Organización donde la familia posee todas las partes y varias generaciones } \\
\text { se involucran en el negocio. }\end{array}$ \\
\hline
\end{tabular}

Fuente. Elaboración propia del autor a partir de Soto Maciel (2013).

Una vez analizado el marco conceptual, histórico y teórico de la empresa familiar, se presenta lo relacionado con la importancia de este tipo de empresas en la economía. 


\subsection{Importancia de la empresa familiar en la economía}

Las empresas familiares tienen gran importancia dentro de la realidad socioeconómica de cualquier país, ya que se constituyen en un agente activo en la regeneración de la estructura económica regional (López, 2003). Por esta razón, la literatura sobre la empresa familiar es muy abundante, fruto de la preocupación tanto de las autoridades públicas como del ámbito académico. Así, por un lado, se habla del gran impacto de las empresas familiares en la creación de riqueza y de empleo, y por otro, se tratan sus importantes fortalezas - elevado compromiso y dedicación, identidad de p.232 valores familiares, transmisión en el tiempo de conocimientos y valores, mayor reinversión de los beneficios y mayor responsabilidad social empresarial-; y debilidades -riesgo de confusión entre cultura e intereses de la familia, rigidez y conservadurismo, autofinanciación y sucesión mal preparada-(Barroso, 2014).

Sin embargo, las fortalezas de las empresas familiares se pueden potencializar y las debilidades superar, a partir de alianzas estratégicas y acuerdos de cooperación que les permitan enfrentarse a la globalización y las transforme en prenda de garantía de su continuidad en el mercado. Muchas de las empresas familiares requieren acuerdos de cooperación para acceder a otros mercados (Lozano, 2003; Fernández, 1996, en Bañegil Palacios, Barroso Martínez y Tato Jiménez, 2011). Las alianzas estratégicas son, por tanto, el camino eficaz para el proceso de internacionalización, pues les permite compartir y encontrar recursos financieros, tecnológicos, humanos, organizativos y relacionales. En otros términos, las alianzas estratégicas ofrecen la posibilidad a las empresas familiares de mejorar su posición competitiva e incrementar sus recursos y capacidades, lo que supondría asegurar la continuidad de la empresa familiar a través de las generaciones.

La continuidad generacional de la empresa es el tópico que guía las líneas siguientes de este documento, donde se analizarán las estructuras de gobierno de las empresas familiares y el problema de la sucesión o su continuidad.

\section{Estructuras de gobierno en la empresa familiar}

\begin{abstract}
Tl gobierno corporativo se refiere a las estructuras y procesos que direccionan y controlan las compañías. Se ocupa de las relaciones entre la alta gerencia, la junta directiva, los accionistas controladores, los accionistas minoritarios y otras partes intere-sadas, a partir de la clara definición de los roles, los deberes, los derechos y las expectativas de cada uno de los órganos de la compañía (IFC, Banco Mundial, 2011).
\end{abstract}

Los órganos más importantes del gobierno corporativo de la empresa familiar son: la asamblea familiar, y el consejo de familia. El primero, es un órgano de carácter informativo y no decisorio, que aglutina a todos los miembros de la familia, a partir de una cierta edad, trabajen o no en la empresa y sean o no propietarios de esta (Galve, 2002, en (Lozano Posso, 2000). El consejo de familia es un órgano de carácter decisorio en cuyo seno se 
discuten tanto los problemas del presente como proyectos de futuro de la familia empresaria. Este órgano contribuye a fortalecer y mantener activos los valores y la historia de la familia, preservando su unidad y armonía (Blumentritt, Keyt \& Astrachan, 2007, en Lozano Posso, 2000).

Ahora bien, las empresas familiares suelen ser más complejas en términos de gobierno que sus contrapartes, debido al agregado de una nueva variable: la familia. Agregar las emociones y los asuntos de la familia a la empresa aumenta la complejidad de los temas corporativos. A diferencia de otros tipos de empresas, los miembros de la familia cumplen distintos papeles dentro de su empresa, lo cual, en muchos casos, puede llevar a la desalineación de los incentivos entre todos sus miembros. En una típica empresa no familiar, cualquier individuo involucrado puede ser un empleado, un ejecutivo, un dueño, un director o alguna combinación de estos papeles.

Sin embargo, en una empresa, propiedad de una familia, un individuo puede tener múltiples papeles y responsabilidades, asociados con diferentes incentivos (IFC- Banco Mundial, 2011). Así, los dueños o accionistas en la empresa familiar tienen varios papeles y motivaciones que pueden llevar a opiniones encontradas. Por ejemplo, una decisión de reinvertir ganancias en la compañía en vez de distribuirlas

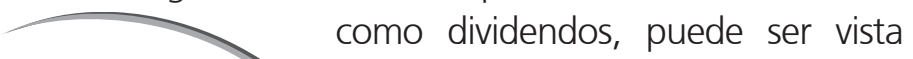
de forma diferente por los diversos $\longrightarrow$ dueños, dependiendo de sus otros p.232 roles en la empresa. Un dueño que trabaja en la empresa familiar podría no objetar una decisión de este tipo, ya que está recibiendo un salario de la compañía. No obstante, esta situación se vería de forma distinta desde la perspectiva de un dueño que no trabaja en la empresa y depende de los dividendos como su fuente principal de ingresos.
Algunos de los papeles que un dueño en una empresa familiar puede tener son: dueño solamente, dueño/ejecutivo, dueño/miembro de la familia, dueño/miembro de la familia/ejecutivo, dueño/director, dueño/miembro de la familia/director, dueño/miembro de la familia/ director/ejecutivo (IFC- Banco Mundial, 2011).

Por su parte, los ejecutivos o alta gerencia de las empresas familiares también tienen distintas motivaciones, dependiendo de sus otros papeles dentro de la empresa. Un tema frecuente en esta área es el tratamiento desigual entre ejecutivos familiares y no familiares. En muchas empresas, algunos o todos los puestos de alta gerencia están reservados estrictamente para miembros de la familia. Esto podría afectar negativamente la motivación y el desempeño de ejecutivos no familiares, ya que saben con certeza que, no importa cuán arduamente trabajen, nunca integrarán la alta gerencia de la empresa. En consecuencia, a muchas empresas familiares les cuesta atraer y retener a ejecutivos talentosos que no pertenecen al núcleo (IFC- Banco Mundial, 2011). En la misma línea, la membresía de la junta directiva es reservada, en la mayoría de las empresas familiares, a quienes toman la mayoría de las decisiones, pues sus puntos de vista contradictorios pueden llevar a conflictos importantes en la junta directiva y afectar negativamente su forma de funcionamiento (IFC- Banco Mundial, 2011).

Lo anterior sugiere que la mala definición de la propiedad y del gobierno corporativo conduce a las empresas familiares hacia dos problemas principales: el primero, es la confusión entre patrimonio empresarial y familiar (Cole, 2000; Kellermanns y Eddleston, 2004) en IFC- Banco Mundial, 2011); y el segundo, es la transmisión del poder y liderazgo de una generación a otra, es decir, la sucesión (Le Breton-Miller, Miller y Steier, 2004; Royer, Simons, Boyd \& Rafferty, 2008, en IFC- Banco Mundial, 2011). 
Estos temas han sido tratados por numerosos autores e investigadores de la materia, y todos llegan a un consenso al identificar la sucesión como una de las principales causas de mortandad en la mayoría de este tipo de negocios (IFC-Banco Mundial, 2011). Ayup, De la Garza y Banda (2008), exponen que una característica natural de los negocios familiares es la propiedad concentrada en los miembros de la familia. La propiedad es considerada como un recurso estratégicamente relevante.

Cuesta (2000, en Castrillo y San Martín, (2007), propone que la implicación familiar en la propiedad y dirección del negocio proporciona ciertas ventajas, ya que una empresa donde coinciden propietarios y directivos puede ser más eficiente debido a que la propiedad familiar permite la convergencia de intereses entre dirección y accionistas, lo que lleva a la maximización del valor de la empresa. No obstante, también puede hablarse de la hipótesis de atrincheramiento donde la propiedad crea incentivos para que los grandes accionistas expropien riqueza de los pequeños (Fama y Jensen, 1983; Shleifer \& Vishny, 1997 en Castrillo y San Martín, 2007). La estructura de gobierno de las empresas y la propiedad de estas, conlleva al problema de sucesión.

\section{El problema de la sucesión o continuidad en las empresas familiares}

T a sucesión, en el ámbito de la teoría de Lla empresa, se conoce como: «el abuelo la funda, los hijos la debilitan y los nietos la entierran» (Le Breton-Miller, Miller \& Steier, 2004; Royer, Simons, Boyd \& Rafferty, 2008 en Soto Maciel, 2013; González, 2010).

Existe una coincidencia unánime de los expertos al señalar que el principal inconveniente de las empresas familiares radica en la dificultad de mantener la continuidad a lo largo de distintas generaciones. De esta manera, la planificación de la sucesión consiste en establecer una estrategia hereditaria en la que entran en juego las reglas de parentesco. El matrimonio unifica, la herencia separa, la propiedad permanece. Dentro del marco legal vigente, cada empresa familiar diseña una estrategia hereditaria particular que no puede ser prevista de antemano. Por lo general, en la mayoría de la empresas familiares, la falta de preparación de las generaciones sucesivas para manejar las demandas de una empresa creciente y una familia mucho mayor, genera una gran crisis (Soto Maciel, 2013).

Navarrete (2008, en Soto Maciel, 2013), analiza los factores de carácter familiar, empresarial y de propiedad, que contribuyen a explicar la continuidad de las empresas familiares. Entre los factores de carácter familiar, la autora incluye la influencia de ciertas características de la relación familiar, como la fuerza de la relación en términos de interdependencia, cohesión y adaptabilidad. Entre los factores empresariales menciona la importancia del tiempo que la empresa ha sido propiedad familiar, la manera en que la familia ha participado en su dirección, así como el desarrollo del sucesor y su vinculación con la dinámica empresarial. Entre los principales obstáculos del proceso de transición generacional, esta misma autora reconoce en el propietario-fundador la resistencia para transferir el poder y el desinterés de los miembros de la familia para participar activamente en cuestiones estratégicas, así como la falta de planificación del proceso de sucesión. 
El tema central de los trabajos de Navarrete (2009), es el análisis de la relación existente entre la implicación familiar y la transición generacional. De los resultados, esta autora afirma que el nexo familiar constituye uno de los principales factores que contribuyen a la continuidad de la empresa. La implicación familiar en la continuidad se vincula a través de los valores, los sueños de vida, los patrones de conducta y las interacciones entre sus integrantes. La autora, concluye reconociendo que la funcionalidad de las estructuras de dirección, propiedad y operación de la empresa dependen del reconocimiento de intereses y metas específicas de cada uno de los dos subsistemas interrelacionados: familia-empresa y empresa-familia.

Barroso (2014), plantea, que a medida que transcurre el tiempo y se transforman las relaciones familiares, es posible que sea necesario modificar el estilo y las claves de gestión dado que aquello que funcionaba en un determinado momento del ciclo de vida, puede ser mejorado en otro momento, por lo que sugiere que la clave está en conseguir una adecuación entre el ciclo vital de la empresa y la gestión óptima en cada ciclo.

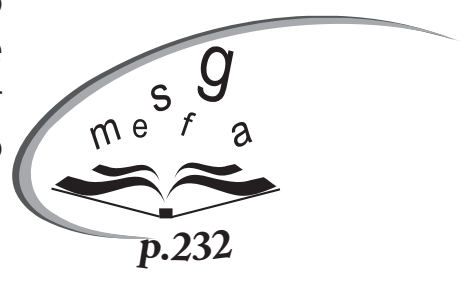

Además de la sucesión de la propiedad, otro de los factores que afectan la falta de continuidad es el escaso nivel de emprendimiento y la falta de gestión profesional. Para McCann, León-Guerrero \& Haley (2011, en Bañegil Palacios, Barroso Martínez \& Tato Jiménez, 2011), profesionalizar el manejo de la empresa es dejar de lado los métodos intuitivos de gestión familiar y adoptar la planificación y el control del crecimiento aplicando métodos estratégicos. La profesionalización en las empresas familiares es la contratación de directivos profesionales no pertenecientes a la familia, que aporten su experiencia y formación en el área concreta que se desea potenciar (Barroso, 2014).

\section{Conclusiones}

E II presente documento delineó de manera suscinta los principales elementos que contextualizan y condicionan la temática de las empresas familiares como agentes claves dentro de los nuevos procesos de globalización de la economía, debido a su rol como generadoras de empleos y procesos de emprendimiento. Este tipo de compañías se constituyen, entonces, en motores de valor agregado y crecimiento.

No obstante, a pesar de las enormes fortalezas de las empresas familiares, su mayor debilidad radica en la dificultad para lograr continuidad generacional, ya que los miembros familiares, sucesores del fundador, por lo general, no tienen el espíritu de su antecesor y tienden a confundir las funciones de la familia con las de la organización. En este sentido, el gobierno corporativo debe servir como ente mediador de estas dificultades y garantizar la transparencia en la gestión empresarial.

Adicionalmente, los procesos de internacionalización y profesionalización de los recursos humanos, administrativos y financieros de las empresas familiares, deben contribuir a la dinamización y sostenibilidad de las empresas familiares. 


\section{Referencias bibliográficas}

Ayup, J., De la Garza, M. y Banda, S. (2008). La empresa familiar en el contexto de la ventaja competitiva. La franquicia como alternativa de crecimiento. Congreso internacional de análisis organizacional, Nuevo Vallarta.

Bañegil Palacios, T., Barroso Martínez, A. y Tato Jiménez, J. (2011). Profesionalizarse, emprender y aliarse para que la empresa familiar continúe. Revista de Empresa Familiar, 1(2), 27-41.

Barroso, A. (2014). Por qué desaparecen las empresas familiares extremeñas. Revista digital para estudiantes de Geografía $y$ Ciencias Sociales, 5(60).

Castrillo, L. y San Martín, J. (2007). La propiedad familiar como mecanismo de gobierno disciplinador de la dirección en las empresas mexicanas. Una evidencia empírica. Contaduría y Administración (222).

González, R. (2010). La continuidad de la empresa familiar. Anuario Jurídico y Económico Escurialense, 401-410.
IFC-Banco Mundial. (2011). Manual IFC de gobierno de empresas familiares. Washington D.C.: Coporación Financiera Internacional (IFC), Banco Mundial.

López, G. (2003). Estudio de la empresa familiar en Rosario. Una aproximación etnográfica. Invenio. Universidad del Centro, 6(11), 91100.

Lozano Posso, M. (2000). El protocolo en las empresas de propiedad familiar. Estudios Gerenciales. Universidad ICESI (74), 49-67.

Soto Maciel, A. (2013). La empresa familiar en México. Situación actual de la investigación. Contaduría y Administración. Universidad Nacional Autónoma de México, 58(2), 135-171.

Steckerl, V. (2006). Modelo explicativo de una empresa familiar que relaciona valores del fundador, cultura organizacional y orientación del mercado. Pensamiento y Gestión (20), 194-215. 
a.

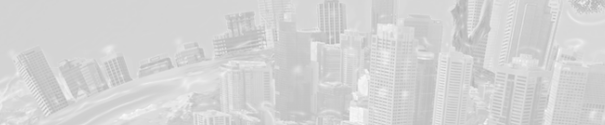

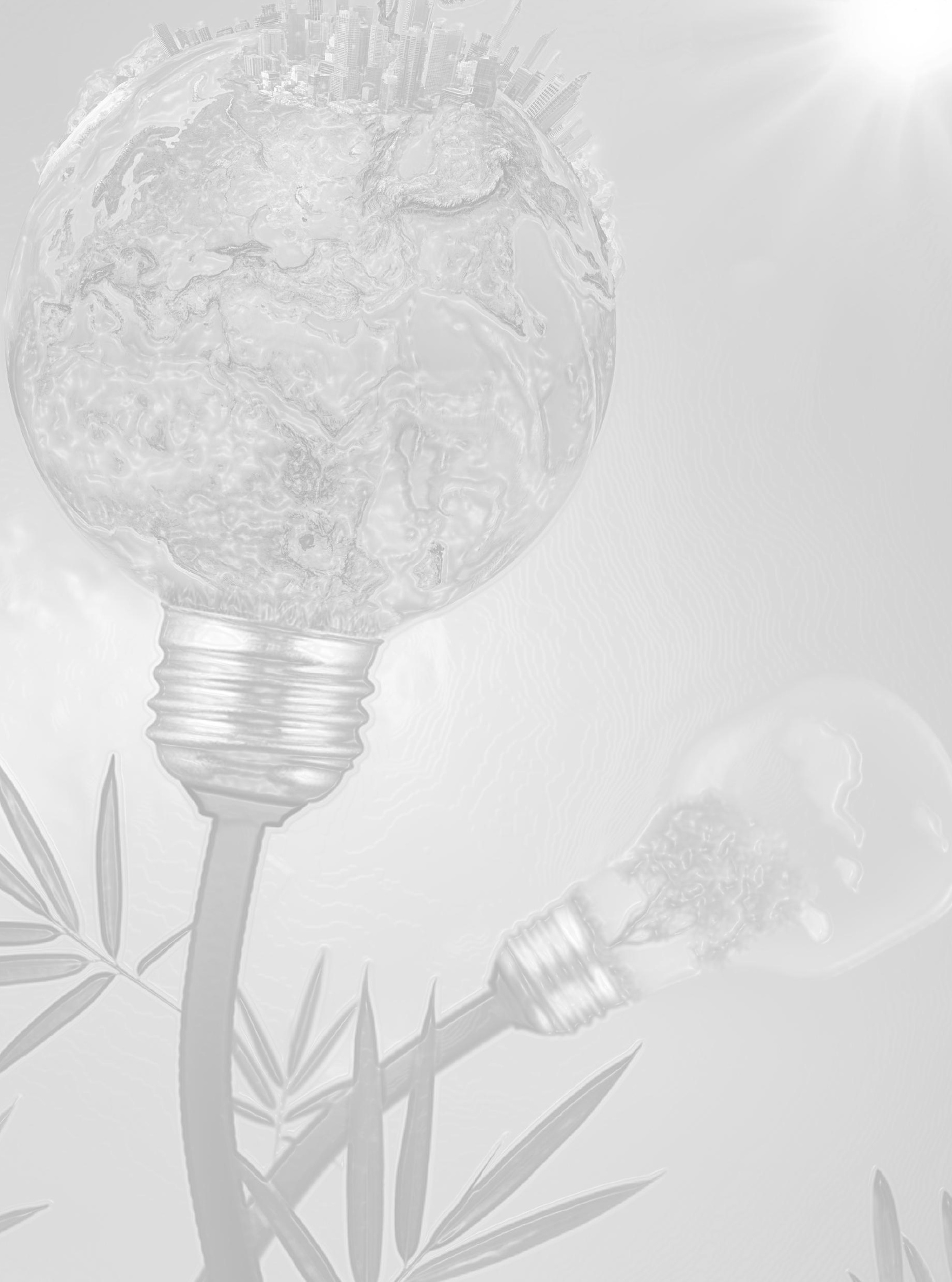

